\title{
Public Relations als Differenzmanagement von externer Kontextsteuerung und unternehmerischer Selbststeuerung*
}

\section{Olaf Hoffjann}

Public Relations (PR) hat ibre Wurzeln als kommunikationswissenschaftliches Forschungsthema als Quelle des Journalismus bzw. als einer von mebreren Akteuren öffentlicher Kommunikation. Die Wurzeln in der Kommunikatorforschung erklären, warum PR bis heute oft reduziert wird auf die Rolle als externer Kommunikator von Organisationen. Die Fokussierung fübrte zu der geringen Zabl an Forschungsarbeiten jenseits der Kommunikatorrolle, vor allem aber zu einem eingeschränkten Verständnis von PR in der Theoriebildung: Hier werden Aspekte wie die Selbstdarstellungs- bzw. Thematisierungsleistung oft überschätzt, wäbrend insbesondere die Reflexionsleistung und Fragen interner Einflussnahme von PR vernachlässigt werden. In dem Beitrag wird auf einer systemtheoretischen Basis vorgeschlagen, unternebmerische Selbststeuerung, also die Beeinflussung der Unternebmenspolitik, als das „Pendant“ zur externen Kontextsteuerung, also der Selbstdarstellungsleistung, zu modellieren. Im Weiteren wird dafür plädiert, PR als Differenzmanagement von externer Kontextsteuerung und unternehmerischer Selbststeuerung zu verstehen, wobei die Kontextstenerung stets die präferierte Seite sein wird.

Schlagwörter: Public Relations, Kommunikatorforschung, Systemtheorie, Steuerungstheorie, Management

Der Stand der PR-Theoriebildung wird trotz unbestrittener Fortschritte bis heute selbst von Fachvertretern kritisch bewertet. So konstatiert Ulrike Röttger: „Public Relations wurde von der deutschsprachigen Kommunikationswissenschaft spät entdeckt, lange Zeit nur wenig erforscht und bis heute in großen Teilen einseitig wahrgenommen - dieser Dreiklang prägte und prägt die PR-Theoriebildung bis heute.“ (Röttger 22009: 9) Die einseitige Wahrnehmung bezieht sich insbesondere auf die Kommunikatorforschung, auf die sich die PR-Forschung bis heute oft selbst beschränkt. In der Kommunikatorforschung wird PR hinsichtlich ihres Beitrags zur Herstellung öffentlicher Kommunikation untersucht. Diese Wurzeln in der Kommunikatorforschung erklären, warum PR bis heute oft reduziert wird auf die Rolle als externer Kommunikator. Die Fokussierung führte zu der geringen Zahl an Arbeiten jenseits der externen Kommunikatorrolle, vor allem aber zu einem eingeschränkten Verständnis von PR in der Theoriebildung: Phänomene jenseits der Kommunikatorrolle bzw. der Selbstdarstellungsleistung finden bis heute insbesondere in der deutschen PR-Forschung wenig Berücksichtigung. Während Reflexion mittlerweile schon häufiger als Leistung der PR genannt wird (z. B. Kussin 2006, 22009; Jarren/Röttger 22009), sind zwei Fragen hingegen selten gestellt worden: Wie kann PR als interner Berater Veränderungen in ihrer eigenen Organisation bewirken? In welchem Verhältnis stehen die Beratungs- und Selbstdarstellungsleistung zueinander? Die Vernachlässigung der internen Seite von PR, so die zu erläuternde These, führt zu einem unzureichenden theoretischen Verständnis von PR, weil die externe Darstellungsleistung als alternativlos dargestellt wird.

* Der Autor dankt Juliana Raupp und Hans-Jürgen Arlt für kritische und konstruktive Anregungen. 
Daher wird in dem Beitrag auf einer systemtheoretischen Basis vorgeschlagen, unternehmerische Selbststeuerung, also die interne Beratungsleistung von PR, als das „Pendant“ zur externen Kontextsteuerung, also der Darstellungsleistung, zu modellieren. In einer solchen Sichtweise sind unternehmerische Selbststeuerung und externe Kontextsteuerung bei der Legitimation des Unternehmens funktional äquivalente Strategien, die zudem einander bedingen. PR betreibt das Differenzmanagement von externer Kontextsteuerung und unternehmerischer Selbststeuerung. Bevor dieser neue Ansatz ausgeführt wird, soll zunächst die Relevanz der externen Selbstdarstellungsleistung und der internen Beratungsleistung in der PR-Forschung diskutiert werden.

\section{Selbstdarstellungsleistung und Beratungsleistung in der PR-Forschung}

PR hat ihre Wurzeln als kommunikationswissenschaftliches Forschungsthema als Quelle des Journalismus bzw. als einer von mehreren Akteuren öffentlicher Kommunikation. So ist es auch kein Zufall, dass die PR bzw. Öffentlichkeitsarbeit in Deutschland ihren Durchbruch als ernst zu nehmendes Forschungsfeld Barbara Baerns’ erstmals 1985 erschienener Arbeit „Öffentlichkeitsarbeit oder Journalismus?“ (Baerns 21991) zu verdanken hat. Elf Jahre später resümierte Wolfgang Langenbucher bei der DGPuK-Jahrestagung 1996 in Leipzig: „Public Relations sind die Verberuflichung von gesellschaftlichen Kommunikatorrollen. Gewiss wäre es theoretisch höchst ergiebig, wenn man PRbezogene Forschungsergebnisse in eine Kommunikator-Begrifflichkeit übersetzen würde." (Langenbucher 1997: 31) Damit beendete er einerseits die Phase der Etablierung der $\mathrm{PR}$ als kommunikationswissenschaftliches Forschungsthema, $\mathrm{zu}$ dem bis heute eine Vielzahl an empirischen und theoretischen Arbeiten erschienen sind. Andererseits zeigt das Zitat einmal mehr, was Ulrike Röttger später als die einseitige Wahrnehmung der PR bezeichnet hat.

Wenn PR aus der Perspektive der Kommunikatorforschung betrachtet wird, wird ihr Beitrag zur Herstellung der Öffentlichkeit beobachtet. Daraus folgt die Selbstdarstellungsleistung bzw. eine externe Thematisierungsleistung von PR, die in vielen Ansätzen zur PR-Theorie in unterschiedlichem Ausmaß eine dominierende Rolle einnimmt. Beispielhaft genannt seien hier die Konstruktion kontingenter Images (vgl. Merten/Westerbarkey 1994: 210), die Mitgestaltung der öffentlichen Informations- und Interpretationsprozesse (vgl. Rolke 1999: 441) und die Herstellung und Bereitstellung durchsetzungsfähiger Themen (Ronneberger/Rühl 1992: 252). Insbesondere der Ansatz von Ronneberger/Rühl (1992) zeigt exemplarisch, zu welchen theoretischen Problemen es führen kann, wenn die Thematisierungsleistung sogar zur zentralen Funktion erhoben wird und PR damit darauf reduziert wird. Die Thematisierungsfunktion wird hier einerseits nicht dem strategischen Charakter der PR gerecht; damit bleibt offen, worin sich PR-Thematisierungen von journalistischen Thematisierungen unterscheiden. Da andererseits die „interne“ Seite der PR weitgehend unberücksichtigt bleibt, lassen die Autoren offen, wofür Organisationen durchsetzungsfähige Themen benötigen.

Im Gegensatz zur externen Selbstdarstellungsleistung der PR ist die Forschungslage zur internen Beratungsleistung insgesamt als eher übersichtlich zu bezeichnen. Während diese internen Leistungen in der deutschen PR-Forschung zwar erkannt, aber kaum zentrale Bausteine vorliegender PR-Theorien sind, nehmen sie in der US-amerikanischen Forschung zwar durchaus eine zentrale Position ein, leiden allerdings unter ihrer normativen Perspektive.

In weiten Teilen der US-amerikanischen PR-Forschung nehmen die externe Selbstdarstellungsleistung und die interne Beratungsleistung traditionell eine gleichberechtigte Stellung ein. Die Definition von Long/Hazleton (1993: 227) steht hier stellvertretend für 
viele: „PR is a communication function of management through which organizations adapt to, alter or maintain their environment for the purpose of achieving organizational goals“ (vgl. z. B. ähnlich Cutlip/Center/Broom 61985: 4; Everett 2000: 314). PR wird hier u. a. als Teil des adaptive subsystems eines Unternehmens verstanden, in dem Unternehmen sich an ihre Umwelt anpassen können (vgl. Cutlip/Center/Broom 61985: 187; Dozier/Grunig 1992: 397). Entsprechend relevant sind Fragen nach der Nähe und dem Zugang zur Unternehmensleitung bzw. zur „dominant coalition“ (Dozier/Grunig/ Grunig 1995: 78), nach deren Unterstützung und nach den hierfür notwendigen Management-Kenntnissen (vgl. ebd.).

Die großen Auswirkungen dieses theoretischen Verständnisses von PR auf die weitere Forschung zeigt exemplarisch das Exzellenz-Forschungsprogramm von James E. Grunig et al. (z. B. Dozier/Grunig/Grunig 1995). So wird solche PR als exzellent bezeichnet, die statt einer asymmetrischen PR eine symmetrische PR betreibe, die einerseits extern als Anwalt der Unternehmensinteressen auftrete, andererseits als „advocates of the publics' interests in discussions with the organisation's strategic planners and decision makers“ (Dozier/Grunig/Grunig 1995: 13). Interne und externe Leistungen von PR werden hier also als gleichberechtigt modelliert. Das Äquivalent zum „two-way symmetrical model“ von Grunig et al. ist bei Cutlip/Center/Broom (61985: 193) in ihrem kybernetischen Ansatz das offene System, das sie dem geschlossenen System PR gegenüberstellen. „The open systems approach puts public relations squarely in the middle of bringing about changes both in the environment and in the organization itself as a result of environmental inputs."

Die große Bedeutung der internen Beratungsleistung ist u. a. aus der Managementorientierung weiter Teile der amerikanischen PR-Forschung zu erklären. Damit hängt zugleich die große Schwäche dieser Arbeiten eng zusammen: ihre normative Perspektive. Dieser normative Anspruch äußert sich in zweifacher Hinsicht. Bereits die Bezeichnung „exzellent“ für symmetrische PR macht die Qualitätsvorstellungen von Grunig et al. deutlich: „We believe, however, that the two-way symmetrical model should be the normative model for public relations - that it describes how excellent public relations should be practiced.“ (Grunig/Grunig 1992: 291) Zudem nehmen sowohl der Ansatz des „two-way symmetrical model“ von Grunig et al. als auch des „open systems“ von Cutlip et al. für sich in Anspruch, eine moralisch „gute“ PR zu gewährleisten. Was bei Grunig et al. die Win-Win-Zone von gesellschaftlichen und organisationalen Interessen ist (vgl. Dozier/Grunig/Grunig 1995: 48), formulieren Cutlip/Center/Broom wie folgt: „The effects sought in this model are maintenance or achievement of goals that are in the organization's and the publics' mutual interests. " (Cutlip/Center/Broom 61985: 196) In einer jüngeren Veröffentlichung konstatieren Grunig und Grunig gar, dass dieses PRVerständnis eine Voraussetzung dafür sei, „that societies around the world need to became more harmonious“ (Grunig/Grunig 2008: 342). Spätestens hier scheint in der PRForschung einmal mehr die Grenze von PR für PR überschritten worden zu sein.

Von einer Überschätzung der externen Darstellungsleistung und einer Unterschätzung der internen Beratungsleistung kann zumindest in der amerikanischen PR-Forschung nicht gesprochen werden. PR wird hier als Antreiber organisationaler Veränderungen betrachtet. Die großen Stärken der Ansätze von Grunig et al. und von Cutlip et al. sind darin zu sehen, dass sie PR als Management-Funktion verorten und die Beziehungen von PR zur Unternehmensleitung und ihren Einfluss thematisieren. Als Problem ist die normative Aufladung anzusehen (vgl. auch Kunczik ${ }^{42002:}$ 217). PR wird idealisiert, wenn als Motiv zur Änderung der Unternehmenspolitik insbesondere moralische Gründe angesehen werden. Aus einer deskriptiven Perspektive erscheint hingegen die 
Frage viel spannender, welche Folgen es für die PR bzw. für das Unternehmen haben kann, wenn es die interne Beratungsleistung nicht gäbe.

In der deutschen PR-Forschung sind Hinweise zur Selbstbeobachtung und Reflexion durch PR ebenfalls seit jeher zu finden. Diese Leistung wird als Frühwarnsystem bzw. „window-in“ (vgl. Beger/Gärtner/Mathes 1989: 37), als Analyse- bzw. Beratungsleistung (Szyszka 2004: 165), Feedbackfunktion (Röttger/Hoffmann/Jarren 2003: 49) oder als Reflexionsleistung (Jarren/Röttger 22009, Kussin 22009) bezeichnet. So unterschiedlich die Begriffe sein mögen, so haben sie gemeinsam, dass sie darin eine zusätzliche Leistung der PR erkennen, bei der „auf der Basis systematischer Umweltbeobachtung legitimations- bzw. organisationsrelevante Informationen aus der Organisationsumwelt in die organisationale Systemreproduktion“ eingespeist werden (Jarren/Röttger 22009: 44). PR-Reflexionen unterscheiden sich von anderen Reflexionen z. B. eines Unternehmens u. a. dadurch, dass sie die Öffentlichkeit bzw. gesellschaftliche Teile jenseits des Marktes beobachten (vgl. Kussin 22009: 124).

So sehr Einigkeit darin besteht, dass PR als organisationale Grenzstelle die Umwelt beobachtet und damit auf mögliche Risiken hinweist, so selten wird diese Leistung in der deutschen PR-Forschung ausführlich erläutert oder nimmt gar eine zentrale Stelle des theoretischen Verständnisses von PR ein (z. B. Zerfaß 22004: 298). Daher lassen sich in der deutschen PR-Forschung nur vereinzelt Hinweise darauf finden, welche Relevanz diese Reflexionen für die gesamte Organisation haben, und insbesondere dazu, wie PR diese Reflexionen z. B. gegenüber der Unternehmensleitung kommuniziert und durchsetzt.

In einer systemtheoretischen Beschreibung modelliert Matthias Kussin (2006, 22009) PR in Anlehnung an Kieserling (vgl. 2005: 83) als Reflexionszentren, die sich insbesondere in komplexen und ausdifferenzierten Organisationen für die Konstruktion und Reproduktion konsistenter Einheitsbeschreibungen als unverzichtbar erweisen können. Diese Beobachtungsleistungen sind einerseits die Grundlage für die externe Kommunikatorleistung, haben „Ausstrahlungseffekte in weitere Organisationsbereiche“ (Kussin 22009: 129) und eröffnen „Orientierungsgesichtspunkte für die fortlaufende Entscheidungsproduktion“ (ebd.). Kussin bietet damit wichtige Hinweise für die Notwendigkeit und die Bedingungen der Reflexionsleistung. Offen bleiben jedoch das (konkurrierende) Verhältnis der PR zu anderen unternehmerischen Reflexionszentren, die Kieserling benennt (vgl. 2005: 82ff.), sowie eine weitergehende Analyse der Beziehungen zur Unternehmensleitung sowie zur Selbstdarstellungsleistung.

Diese Aspekte werden auch von solchen Autoren nur wenig thematisiert, die wie die genannten amerikanischen Autoren die interne Beratungsleistung als gleichberechtigte Leistung neben der Selbstdarstellungsleistung modellieren. Ulrike Röttger weist der PR als zweite Wirkungsrichtung zu, dass PR organisationsintern Einfluss auf die Organisationspolitik nehme, um Legitimationspotenziale optimal zu nutzen (vgl. Röttger 2008: 75, 2000: 88). Ein ausgearbeiteter theoretischer Ansatz zum Verhältnis zur organisationsexternen Wirkungsrichtung fehlt aber ebenso wie bei Peter Szyszka, bei dem PR für externe Interventionsprogramme anschlussfähige Entscheidungen der Organisation im Zweifel herbeiführen muss (vgl. Szyszka 22009: 148).

Die Unterschätzung der internen Beratungsleistung in der deutschen PR-Forschung überrascht insbesondere, weil die amerikanische PR-Forschung in Deutschland in hohem Maße - wenn auch zumeist kritisch - wahrgenommen wird. Zudem hat sich bereits Albert Oeckl in seiner normativ und praktizistisch angelegten „PR-Praxis“ (1976) ausführlich mit dieser Frage auseinandergesetzt, als er Öffentlichkeitsarbeit als Summe von Information, Anpassung und Integration definierte (Oeckl 1976: 19) und für eine er- 
folgreiche Öffentlichkeitsarbeit eine „vertretbare Selbstkorrektur“ (ebd.: 20) von Unternehmen einforderte.

Die Diskussion des Forschungsstandes hat gezeigt, dass sowohl die deutsche als auch die amerikanische PR-Forschung zumindest „auf einem Auge blind“ sind. Die deutsche PR-Forschung wird von Fragen der externen Selbstdarstellungsleistung und damit der Kommunikatorrolle dominiert. Damit geraten aber alternative Lösungsstrategien wie die interne Beratungsleistung aus dem Blick. Zudem wird damit das zu lösende Problem von PR meist auf ein Kommunikationsproblem reduziert. Die amerikanische PR-Forschung berücksichtigt zwar auch die interne Beratungsleistung, neigt aber zu einer normativen Verklärung, wenn sie Veränderungen der Organisationspolitik vor allem mit ethischen Motiven erklärt.

Aus dem Forschungsstand ergeben sich insbesondere die folgenden Fragen:

- Externe Selbstdarstellungsleistung vs. interne Beratungsleistung: In welchem Verhältnis stehen die interne Beratungsleistung und die externe Selbstdarstellungsleistung zueinander? In welchen Fällen dominiert eine der beiden Leistungen? Lassen sich Mischtypen beobachten?

- Interne PR-Beratungsleistungen vs. Unternebmensleitung: Wenn PR ein organisationales Reflexionszentrum ist, stellt sich die Frage, wie die Selbstbeschreibungen der PR organisationsweite Verbindlichkeit erlangen (vgl. Kieserling 2005). Was erhöht bzw. mindert die Chancen? Mit welchen anderen Selbstbeschreibungen bzw. Interessen konkurriert PR intern? Welche Konsequenzen ergeben sich für die PR und für das Unternehmen, wenn die internen Beratungsleistungen von PR nicht erbracht werden bzw. von der Unternehmensleitung nicht nachgefragt werden?

Damit diese Fragen beantwortet werden können, braucht es zunächst ein theoretisches Verständnis von PR, das die Selbstdarstellungsleistung bzw. die Kommunikatorrolle nicht als Ausgangspunkt nimmt, sondern zunächst fragt, was der Zweck bzw. - in systemtheoretischer Perspektive - das zu lösende Problem von PR ist. Hier soll insbesondere die Frage beantwortet werden: Welche Probleme löst PR? Dieser Beobachtungsstandpunkt ist gewissermaßen eine Stufe über der Selbstdarstellungsleistung, setzt diese damit kontingent und macht den Blick frei für funktional äquivalente Strategien. Hier stellt sich dann die Frage: Wie löst PR die Probleme?

Diese Fragen sollen auf einer systemtheoretischen Basis beantwortet werden. Eine solche Perspektive bietet sich einerseits an, weil sich für die zentralen Kategorien der PR, Organisation und Öffentlichkeit, die systemtheoretische Modellierung „längst als dominanter Theorietyp" etabliert hat (Merten 22009: 68) und damit an vorhandene Arbeiten angeschlossen werden kann. Vor allem aber erscheint die systemtheoretische Perspektive der Komplexität des Untersuchungsgegenstandes gerecht zu werden, die schon in der Diskussion des Forschungsstandes deutlich geworden ist. So muss zunächst das zu lösende Problem der PR herausgearbeitet werden; zudem sind die Beziehungen der PR zur Unternehmensleitung und zum Gesamtunternehmen zu beschreiben; schließlich sind zur Erläuterung der Selbstdarstellungsleistung auch Bezugsgruppen sowie die Öffentlichkeit relevant. Zur Beschreibung von Beziehungen zwischen Akteuren bzw. Systemen unterschiedlicher Abstraktionsgrade scheint die systemtheoretische Perspektive besonders geeignet. 


\section{Differenzmanagement von externer Kontextsteuerung und unternehmerischer Selbststeuerung}

Bevor untersucht wird, wie PR Unternehmen legitimiert, wird herauszuarbeiten sein, was das zu lösende Problem und damit die Funktion von PR ist. Public Relations wird in der Literatur in der Regel auf das gesellschaftliche Umfeld eines Unternehmens ${ }^{1}$ hin ausgerichtet. Hier können Unternehmen von vielen Bezugsgruppen abhängig sein - von Zulieferern, von der Politik oder vom Erziehungssystem. Um künftige Handlungsspielräume zu sichern und Konflikte zu vermeiden, sind Unternehmen auf Legitimität angewiesen. Mit Legitimität meint Fuchs-Heinritz, „dass Herrschende, politische Bewegungen und Institutionen aufgrund ihrer Übereinstimmung mit Gesetzen, Verfassungen, Prinzipien oder aufgrund ihrer Leistungsfähigkeit für allgemein anerkannte Ziele akzeptiert, positiv bewertet und für rechtmäßig gehalten werden“ (Fuchs-Heinritz 31994: 396, vgl. Syzszka/Bentele 2005: 592). Unternehmen, die von anderen Bezugsgruppen legitimiert sind, müssen gegenüber diesen nicht mehr jede Entscheidung begründen. Denn Legitimität setzt als Teil der Umwelterwartungen eines Systems Lernwilligkeit voraus, weil sie eine generalisierte Bereitschaft ist, „inhaltlich noch unbestimmte Entscheidungen innerhalb gewisser Toleranzgrenzen hinzunehmen“" (ebd.: 28). Fehlende Legitimität ist damit das zentrale Problem, auf dessen Lösung sich PR spezialisiert hat. Legitimität bzw. Legitimation als zentrale Begriffe der PR besitzen in der PRForschung eine lange Tradition - genannt seien hier nur Ronneberger (1977), Everett (2000), Metzler (2000), Zerfaß (2004), Szyszka (22009) und Jarren/Röttger (22009). Weitere Begriffe wie Akzeptanz (z. B. Kohring/Hug 1997), Vertrauen (z. B. Hundhausen 1951, Oeckl 1964; Kunczik 42002) und Reputation (z. B. Eisenegger 2005), die im Kontext der PR diskutiert werden, erscheinen zur Sicherung künftiger Handlungsspielräume allenfalls als notwendige, keinesfalls aber als hinreichende Voraussetzung.

Die Funktion von Public Relations ist demnach die Legitimation der Organisationsfunktion gegenüber den als relevant eingestuften Bezugsgruppen in der Gesellschaft. Da PR und Legitimation kein Selbstzweck sind, sondern fehlende Legitimität zu einem Kaufboykott oder zur Einreichung einer Klage führen kann, machen sie immer nur Sinn in Relation zum Organisationserfolg. Denn Unternehmen müssen damit rechnen, dass Einzelne ihre ablehnende Haltung durch Kaufboykotte oder den Gang zum Gericht äußern. In systemtheoretischer Argumentation hat PR folglich keine gesellschaftliche Funktion, sondern löst ein organisationales Problem und wird mithin als Subsystem von Unternehmen modelliert. (Vgl. Hoffjann 22007) Diese Problem- und Funktionsbeschreibung reduziert PR nicht auf die Lösung eines „Kommunikationsproblems“ und schafft damit eine Differenzierung zu anderen Phänomenen wie der Absatzkommunikation und der Mitarbeiterkommunikation. Damit ist eine differenzierte Antwort auf die Besonderheit der Probleme, die PR löst, gegeben.

Auf dieser Grundlage kann die Selbstdarstellungsleistung von PR kontingent gesetzt werden. Damit sollen jetzt funktional äquivalente Strategien identifiziert werden, wie PR Unternehmen legitimiert.

1 Obwohl PR nicht nur in Unternehmen, sondern auch in anderen Organisationen wie Kirchen oder Verbänden zu beobachten ist, sind die folgenden Überlegungen weitgehend auf Unternehmen fokussiert, um die systemtheoretisch basierte Argumentation durchgängig mit Beispielen aus dem unternehmerischen Bereich zu konkretisieren. 
Wenn Legitimität u. a. die Vermeidung von Konflikten mit relevanten Bezugsgrup$\operatorname{pen}^{2} \mathrm{zu}$ relevanten Themen voraussetzt, wird PR in der Regel bestrebt sein, konfligierende Erwartungsstrukturen zu erkennen und zu „bearbeiten“. In einem vereinfachten Modell sind hier zwei Situationen vorstellbar: PR trifft entweder auf eine kognitive oder auf eine normative Erwartungshaltung einer Bezugsgruppe. Eine kognitive Erwartungshaltung, also Lernbereitschaft, kann von der Zuschreibung von Legitimität selbst bis hin $\mathrm{zu}$ einer konstruktiv-kritischen Beobachtung eines Unternehmens reichen - eine Bezugsgruppe ist also noch zu überzeugen. Eine normativ-kritische Erwartungshaltung schließt hingegen zunächst die Zuschreibung von Legitimität aus: Die Bezugsgruppe steht dem Unternehmen insgesamt oder bezogen auf ein Thema kritisch gegenüber.

Daraus lassen sich zwei idealtypische Strategieoptionen ableiten, die empirisch in der Regel als Mischtypen zu beobachten sein werden.

- Wenn PR den relevanten Bezugsgruppen eine kognitive Erwartungshaltung unterstellt, kann das Unternehmen an seiner Unternehmenspolitik festhalten und z. B. mittels der externen Kommunikation von Selbstbeschreibungen versuchen (vgl. Kieserling 2005), die Umwelterwartungen dieser Bezugsgruppen gegenüber dem Unternehmen zu ändern und damit das Unternehmen zu legitimieren. Diese Strategieoption entspricht im Wesentlichen der bereits diskutierten Selbstdarstellungsleistung.

- Unterstellt PR bei den relevanten Bezugsgruppen eine normativ-kritische Erwartungshaltung, und hat eine einzelne Unternehmensentscheidung das Potenzial, die Legitimation des gesamten Unternehmens zu gefährden, wird PR die eigene Unternehmenspolitik zu ändern versuchen. Dies entspricht im Wesentlichen der internen Beratungsleistung von PR.

Es ist zu erwarten, dass zumeist beide Strategien gleichzeitig eingesetzt werden - Änderungen der Unternehmenspolitik also von Selbstdarstellungen begleitet werden. In einer solchen Sichtweise managt PR den Einsatz dieser beiden Strategien bzw. die Unterscheidung dieser beiden Strategien. PR entscheidet also in jedem Fall neu, in welchem Ausmaß die externe Darstellungsleistung ausreicht und die interne Beratungsleistung notwendig ist. Damit wird die interne Beratungsleistung nicht zu etwas „Zusätzlichem" von PR. PR ist in einer solchen Sichtweise ohne die interne Beratungsleistung nicht denkbar.

Dieses Differenzmanagement soll im Folgenden in einer systemtheoretischen Perspektive erläutert werden. Dabei sollen die externe Darstellungsleistung und die interne Beratungsleistung steuerungstheoretisch als externe Kontextsteuerung und als unternehmerische Selbststeuerung ${ }^{3}$ modelliert werden.

Die Möglichkeiten der Steuerung sozialer Systeme werden in der Systemtheorie unterschiedlich beurteilt. Während Niklas Luhmann mit seiner „radikal nihilistische[n] Position zur Steuerungsfähigkeit“ (Druwe/Görlitz 1992: 151) skeptisch gegenüber Steuerungskonzepten war, hält Helmut Willke dem entgegen, dass neben interner Selbststeuerung auch die Kontextsteuerung die operative Geschlossenheit von Systemen respektiere (vgl. Willke ${ }^{31995)}$. Denn eine erfolgreiche Kontextsteuerung eines steuernden Systems geht immer einher mit der Selbststeuerung des zu steuernden Systems. Zudem

2 Da jedes Unternehmen eine Vielzahl von Bezugsgruppen hat, die unterschiedliche Ansprüche an das Unternehmen stellen, wird PR diese Bezugsgruppen im Rahmen der Legitimation hierarchisieren. Entscheidend hierfür wird insbesondere das direkte oder indirekte Sanktionspotenzial sein, das einer Bezugsgruppe zugeschrieben wird.

3 Ruß-Mohl hat die Begriffe Außen-Steuerung und Selbststeuerung bereits im Kontext der PR diskutiert, allerdings im Rahmen der Autonomie-Diskussion von PR (vgl. Ruß-Mohl 1992). 
bleiben solche Steuerungsversuche in der Regel auch nicht ohne Folgen für das steuernde System selbst - sind also mit Selbststeuerungen des steuernden Systems verbunden. Im Folgenden werden zunächst die Kontext- und die Selbststeuerungen der PR getrennt erläutert werden, bevor ihr Differenzmanagement durch PR ausgeführt wird.

\subsection{Externe Kontextsteuerung der PR}

Mit dem Konzept der externen Kontextsteuerung soll erklärt werden, wie PR die Unternehmensumwelt bzw. relevante Teile von ihr zu steuern versucht. Zunächst ist das Konzept theoretisch zu modellieren, um anschließend die Fragen nach der „Eigenart“ von PR-Steuerungen zu diskutieren sowie die nach den Möglichkeiten und Grenzen externer Kontextsteuerungen.

In der Kontextsteuerung wird die operative Geschlossenheit respektiert und stattdessen versucht, die Umweltbedingungen eines Systems so zu verändern, dass sich dieses qua Selbststeuerung in die gewünschte Richtung verändert (vgl. Willke 31995). Die Kontextsteuerung setzt folglich in der Umwelt Bedingungen, ,an denen sich das zu steuernde System in seinen eigenen Selektionen orientieren kann und im gelingenden Fall im eigenen Interesse orientieren wird“ (Willke 1997: 141). Kontextsteuerung ist damit die Konditionierung eines Systems und reduziert die Kontingenz von Operationen wie Entscheidungen, ohne sie zu determinieren. Sie erlaubt es folglich dem System, „weiterhin die eigene Melodie zu spielen, nun aber in einem anderen Rhythmus“ (Willke 1996: 191).

Da diese Veränderungen der Kontextbedingungen mithin in der Umwelt eines Systems stattfinden, versteht ein zu steuerndes System diese Außeneinwirkungen nur dann als Informationsangebote, wenn diese in einer Form vorliegen, die nach den Suchschemata des intervenierten Systems Sinn machen (vgl. Willke 21993: 130). Die Erfolgschancen der Kontextsteuerungen hängen folglich im hohen Maße davon ab, wie gut das steuernde System die Strukturen des zu steuernden Systems kennt. Wie aber lernen Systeme etwas über die Strukturen anderer Systeme, wie zum Beispiel deren Selektionskriterien, wenn sie zunächst einmal intransparent füreinander sind und sich in einer „Black-boxInteraktion“ (Willke 1992: 35) befinden? Hierzu tragen insbesondere Erfahrungen aus kontinuierlichen Beziehungen bei-Systeme lernen, was zu erfolgreichen Kontextsteuerungen geführt hat und was nicht. Für die Erfolgschancen von Steuerungsversuchen bedeutet dies: Je öfter Interaktionen stattfinden, desto stärker sind die strukturellen Kopplungen und desto größer ist die Wahrscheinlichkeit, dass die Perturbationen vom zu steuernden System adäquat strukturell abgearbeitet werden (vgl. Druwe/Görlitz 1992: 155f.). Um die Steuerungsanreize möglichst genau ansetzen zu können, ist PR mithin auf ein Mindestmaß an Eigenkomplexität angewiesen (vgl. Nothhaft/Wehmeier 22009: 164f.).

Willke greift mit seinem Konzept der Kontextsteuerung (Willke ${ }^{31995)}$ Ideen der Soziokybernetik auf, in der die Relevanz der Rahmenbindungen für bestimmte Situationen schon lange vorher erkannt und beschrieben wurde (vgl. z. B. Meyer/Rowan 1977). Nothhaft/Wehmeier (22009) sehen in der Soziokybernetik und in der von ihnen ebenfalls so benannten Kontextsteuerung bzw. -kontrolle einen geeigneten Erklärungsansatz für PR-Phänomene: „Vertrauen, Glaubwürdigkeit, Loyalität oder Legitimität lassen sich nicht herstellen, sondern lediglich verdienen und erwerben - wir bauen es nicht, es wächst.“ (Nothhaft/Wehmeier 22009: 163) Einerseits legen Nothhaft/Wehmeier sehr plausibel dar, dass PR zum Beispiel im Gegensatz zur Werbung insbesondere auf indirekte Mechanismen setzt. Andererseits übersehen sie dabei, dass direkte Steuerungsversuche in einer soziokybernetischen genauso wie in einer systemtheoretischen Argu- 
mentation zum Scheitern verurteilt sind. Mit anderen Worten: Kontextsteuerung ist als externer Steuerungsmodus alternativlos. Die Kontextsteuerung allein kann den spezifisch „indirekten“ Charakter von PR-Steuerungsversuchen folglich nicht erklären.

Wie betreibt PR in Unternehmen also Kontextsteuerung? Allgemein formuliert: PR versucht mit der externen Kontextsteuerung, die Umweltbedingungen ausgewählter Bezugsgruppen so zu verändern, dass die Bezugsgruppen unbequeme Entscheidungen akzeptieren bzw. die Unternehmensinteressen bei eigenen Entscheidungen berücksichtigen (vgl. ähnlich Jarren/Röttger 22009, Nothhaft/Wehmeier 22009). Dazu kreiert PR zum Beispiel argumentative „Anreize“, die bei den Bezugsgruppen anschlussfähig sind. Solche „Anreize“ sind Selbstbeschreibungen über die positiven Folgen des eigenen Handelns. Diese Selbstbeschreibungen der PR betonen insbesondere die Relevanz externer Interessen für die Organisation. Damit versucht PR, den Gegensatz von eigensinnigem Verhalten und gesellschaftlichen Interdependenzen aufzulösen. Dazu werden die Rationalitäten bzw. Selektionskriterien der relevanten Bezugsgruppen in den Selbstbeschreibungen berücksichtigt - bei Amnesty International die Frage der Menschenrechte, bei Greenpeace die Relevanz des Naturschutzes usw. PR übernimmt damit in Unternehmen die Rolle eines „Übersetzungsbüros“ (Röttger/Hoffmann/Jarren 2003: 49). All diese Kommunikationen simulieren andere gesellschaftliche Perspektiven, um dem Vorwurf der Eigensinnigkeit entgegenzutreten.

Neben „argumentativen“ Anreizen kann PR auch finanzielle Anreize im Rahmen der Kontextsteuerung einsetzen. ${ }^{4}$ Das Sponsoring von Non-Profit-Organisationen wie Schulen und selbst kritischen Interessenorganisationen zielt einerseits darauf, durch den freiwilligen Verzicht auf Gewinne erneut die Relevanz gesellschaftlicher Werte zu kommunizieren. Andererseits vergrößert PR insbesondere mit dem Sponsoring von unterstützenden Interessenorganisationen oder von örtlichen Sport- und Kulturvereinen seine öffentlichen Unterstützerpotenziale. Insgesamt scheint das Medium Geld aber für die Legitimation nur ein nachgeordnetes Steuerungsmedium zu sein. Geld als Steuerungsressource zur Finanzierung von öffentlichen Kommunikationskampagnen mag zwar in der Regel notwendig sein, die erfolgreiche Legitimation vieler Non-Profit-Organisationen und die erfolglose Legitimation von großen Unternehmen zeigen aber, dass Geld keine hinreichende Voraussetzung ist. Und so dürften die genannten Steuerungsbeispiele mittels des Mediums Geld auch künftig nur eine unterstützende Relevanz besitzen. In der externen Kontextsteuerung dürfte mithin Kommunikation über die positiven Folgen unternehmerischen Handelns die größte Bedeutung behalten.

Im Rahmen der Pressearbeit versucht PR, journalistische Selektionskriterien zu simulieren, indem es Anreize für eine Berichterstattung schafft. Diese Anreize reichen von dem Versprechen der Exklusivität über die Inszenierung von Veranstaltungen und provokante Statements bis hin zum Einsatz prominenter Testimonials bei PR-Anlässen. Damit setzt PR zwar an redaktionellen Programmen an, verletzt aber nicht die redaktionelle Autonomie, sondern schafft Berichterstattungsanlässe in der redaktionellen Umwelt. Angesichts der zunehmenden Ausbreitung und Professionalisierung von PR trifft der Journalismus mittlerweile fast nur noch auf solche „PR-getunten“ Veranstaltungen und Kommunikationsangebote von Unternehmen.

Die beschriebenen Kontextsteuerungen können als direkte bzw. einfache Kontextsteuerung bezeichnet werden. Solche Kontextsteuerungen zielen auf eine direkte Beeinflussung der Systeme. Davon kann die indirekte bzw. doppelte Kontextstenerung unter-

4 Es wird später zu zeigen sein, dass solche Kontextsteuerungen eine unternehmerische Selbststeuerung voraussetzen. 
schieden werden. So stellen die direkten Kontextsteuerungen gegenüber dem Journalismus zugleich eine indirekte bzw. doppelte Kontextsteuerung gegenüber anderen Bezugsgruppen dar. Denn durch die „Bearbeitung“ des Journalismus beeinflusst PR zugleich das Öffentlichkeitssystem (vgl. dazu ausführlich: Görke 1999) und damit weitere relevante Bezugsgruppen. Dies wird im Folgenden zu erläutern sein. Während die Öffentlichkeit und der Journalismus nur ein indirektes Sanktionspotenzial besitzen, weil hier zum Beispiel keine politischen Entscheidungen getroffen werden, ist die Politik für unternehmerische PR eine Bezugsgruppe mit direktem Sanktionspotenzial, weil sie die Handlungsfreiheit durch neue Gesetze einschränken kann. Dennoch haben die Öffentlichkeit und der Journalismus als ihr Leistungssystem eine herausragende Relevanz für die PR, weil sich der Öffentlichkeit wegen ihres intermediären (vgl. Gerhards/Neidhardt 1991: 41) bzw. synchronisierenden (vgl. Görke 1999: 300) Charakters nur wenige gesellschaftliche Teilbereiche „entziehen“ können. Die (beobachteten) Mehrheiten in der Öffentlichkeit beeinflussen also wesentlich die Legitimation eines Unternehmens. Daher dürfte nicht nur die Unternehmens-PR es scheuen, Entscheidungen gegen die (beobachtete) Mehrheit der Öffentlichkeit zu treffen und öffentlich zu kommunizieren, sondern auch ihre kritischen Bezugsgruppen. Wenn es PR also gelingt, mittels einer erfolgreichen Kontextsteuerung gegenüber dem Journalismus weite Teile der Öffentlichkeit für die eigene Position zu gewinnen, werden zum Beispiel andere Unternehmen im eigenen Interesse kaum konträre Positionen öffentlich kommunizieren, wenn sie damit zum Beispiel ihre Glaubwürdigkeit und ihre eigene Legitimität riskieren. Damit betreibt $P R$ eine indirekte bzw. doppelte Kontextsteuerung dieser relevanten Bezugsgruppen über die Öffentlichkeit. Diese doppelte Kontextsteuerung ist der spezifisch „indirekte“ Charakter von PR. Die breite öffentliche Unterstützung eines Unternehmens dürfte eine kritische Bezugsgruppe eher von weiteren Protestaktionen abhalten als „gute Argumente" in direkten Gesprächen.

Hier lassen sich bereits die Möglichkeiten der PR-Kontextsteuerungen erahnen. Zentrale Voraussetzungen hierfür scheinen zu sein, dass Kontextsteuerungen langfristig angelegt sind (Zeitdimension), inhaltliche Widersprüche gegenüber verschiedenen Bezugsgruppen und von unterschiedlichen Kommunikationsstellen des Unternehmens weitgehend vermieden werden und die geschaffenen Anreize anschlussfähig sind (Sachund Sozialdimension). Die Grenzen der Kontextsteuerungen werden beispielsweise in Krisensituationen deutlich, in denen Journalismus deutlich eigeninitiativer und rechercheintensiver arbeitet als in Normalsituationen (vgl. Barth/Donsbach 1992). Steuerungsträume der PR begrenzen neben der operativen Geschlossenheit von Systemen zunehmend auch die konkurrierenden PR-Stellen anderer Unternehmen.

\subsection{Unternebmerische Selbststeuerung}

Während PR mit externer Kontextsteuerung versucht, die Erwartungsstrukturen relevanter Bezugsgruppen zu verändern, versucht sie mit unternehmerischer Selbststeuerung, das Unternehmen zu verändern. Der Bedarf an unternehmerischer Selbststeuerung dürfte besonders groß sein, wenn PR respektive ein Unternehmen auf relevante Bezugsgruppen mit einer normativ-kritischen Erwartungshaltung und einer vermuteten großen öffentlichen Unterstützung treffen. Ein (öffentlicher) Konflikt und damit weitere Legitimationsverluste über diese Bezugsgruppe hinaus sind dann allenfalls nur durch eine veränderte Unternehmenspolitik, also eine unternehmerische Selbststeuerung zu erreichen.

Selbststeuerungen sind untrennbar mit Reflexion verbunden. Reflexion sind Operationen, die sich auf die Identität des Systems beziehen (vgl. Luhmann 1981: 423). „Re- 
flexive Orientierung eines individuellen oder kollektiven Akteurs meint die Fähigkeit zur Empathie, also die Fähigkeit, sich selber in die Rolle anderer Akteure zu versetzen, um aus deren Perspektive die eigene Rolle zu sehen." (Teubner/Willke 1984: 14) Eine solche reflexive Orientierung leistet - wie bereits ausgeführt - u. a. PR als unternehmerisches Reflexionszentrum (vgl. Kussin 2006, 22009). PR reflektiert dabei die Identität des Gesamtunternehmens gegenüber funktionssystemfremden Rationalitäten und sucht manifeste sowie potenzielle Legitimationsrisiken und -chancen.

Diese Reflexionen sind „eine Form der Selbststeuerung, durch welche Teilsysteme ihre eigene Identität thematisieren und genau darauf einstellen, dass in ihrer relevanten Umwelt andere Teilsysteme in Interdependenzbeziehungen agieren und sie selbst für diese anderen Teilsysteme eine brauchbare Umwelt darstellen müssen “ (Teubner/Willke 1984: 14). Die Funktion von PR im Kontext unternehmerischer Selbststeuerung ist es folglich zu gewährleisten, dass Unternehmensentscheidungen auf möglichst geringen Widerstand in der Umwelt treffen - ggf. also Unternehmensentscheidungen so getroffen werden, damit sie auf Zustimmung stoßen. Da solche Selbststeuerungen Legitimität ebenso sichern oder schaffen können wie Kontextsteuerungen, sind Selbststeuerung und Kontextsteuerung funktional äquivalente Strategien zur Legitimation eines Unternehmens.

Nicht Legitimation, sondern Geld ist jedoch die Sprache von Unternehmen. Zudem steht Legitimation in Konkurrenz zu anderen nachgeordneten Problemen in Unternehmen. Die bislang oft vernachlässigte Frage ist folglich, wie PR in einer solchen Konkurrenzsituation Selbststeuerungen eines Unternehmens bewirken kann. Zur Beantwortung dieser Frage muss zwischen der PR-Selbststeuerung und der unternehmerischen Selbststeuerung unterschieden werden.

- PR-Selbststenerungen beziehen sich auf Veränderungen, die in der Autonomie des organisationalen Subsystems PR liegen - zum Beispiel Änderungen, die aus strukturellen Kopplungen mit dem Journalismus resultieren.

- Als unternehmerische Selbststeuerungen hingegen sollen Veränderungen bezeichnet werden, die durch die Unternehmensleitung entschieden werden müssen. Dazu zählen zum Beispiel der Ausstieg aus der Kernenergie, um den Fortbestand des Unternehmens nicht zu gefährden; der Verzicht auf die Versenkung der Ölplattform, um den Absatz nicht zu gefährden; eine Strukturierung und damit höhere Investitionen in CSR-Aktivitäten, um künftige Konflikte mit Stakeholdern zu vermeiden bzw. das Vertrauen in das Unternehmen zu erhöhen; ein Verzicht auf Kinderarbeit in Südamerika, um Diskussionen mit Verbraucherschutzorganisationen zu beenden.

Als Teil- bzw. Subsystem eines Unternehmens kann PR solche Änderungen der Unternehmenspolitik nur empfehlen. Solche empfohlenen Verhaltensänderungen beziehen sich auf Legitimationschancen und -risiken. Eine Unternehmensleitung wird die Folgen einer solchen Entscheidung bzw. die Auswirkungen für das Gesamtunternehmen diskutieren und entscheiden. Nicht selten nehmen Unternehmen bewusst eine Delegitimation in Kauf, um zum Beispiel kurzfristige wirtschaftliche Ziele nicht zu gefährden. Daher wird PR bei solchen Empfehlungen nicht nur legitimationsbezogen, sondern immer aus einer gesamtunternehmerischen Perspektive argumentieren - also insbesondere die monetären Auswirkungen im Blick haben.

Damit ist die unternehmerische Selbststeuerung letztlich eine Kontextsteuerung der PR gegenüber der Unternehmensleitung: Je besser es der PR gelingt, die PR-Risiken in monetäre Risiken zu „übersetzen“ und damit Anschluss an Programme der Unternehmensleitung zu gewinnen, desto größer sind die Erfolgschancen dieser Steuerungsversuche. PR leistet somit eine Anleitung zur Selbststeuerung (vgl. Willke 21993: 130). Die 
internen Steuerungsversuche sind zudem tendenziell umso erfolgreicher, je größer der formale oder informelle Einfluss der PR auf die Unternehmensleitung ist (vgl. Dozier/ Grunig/Grunig 1995: 75ff.).

Das strukturelle Problem zwischen Unternehmensleitung und PR ist, dass insbesondere solche Selbststeuerungsentscheidungen legitimierend wirken können, die wirtschaftlich höchst irrational sind - also ein freiwilliger Verzicht auf einen Teil des Gewinns. Die Investition in umweltschonende Produktionsverfahren, die Verbesserung der Arbeitsbedingungen von Produktionsstätten in Schwellenländern oder der Verzicht auf sogenannte Alkopop-Getränke sind nur einige wenige Beispiele für gleichermaßen legitimationsrelevante wie kostspielige Entscheidungen. Dieser „eingebaute Widerspruch“ macht PR damit zum „schlechten Gewissen“ von Unternehmen. Weil PR zumindest kurzfristig Handlungsspielräume einschränkt, weil PR dabei auf die Unterstützung der Unternehmensleitung angewiesen ist und weil die Empfehlungen von PR in der Regel auch ex post schwer zu quantifizieren sind, setzt dies ein enormes Vertrauen der Unternehmensleitung sowohl in das PR-System als auch in die verantwortlichen Personen voraus. Dies dürfte, so ist zu vermuten, nicht ohne Auswirkungen auf die PRAkteure bleiben. Bei Empfehlungen zu möglichen Änderungen der Unternehmenspolitik dürften sie sich auch die Frage stellen, welche Auswirkungen eine mögliche Ablehnung auf ihre eigene Position hat.

\subsection{Das Management der Unterscheidung von externer Kontextsteuerung und unternebmerischer Selbststeuerung}

PR managt die beiden erläuterten Steuerungsmodi, um das unternehmerische Problem der Legitimation zu bearbeiten. Nachdem bislang die beiden Steuerungsmodi getrennt erläutert wurden, soll nun ihr Verhältnis zueinander analysiert werden.

Zunächst einmal bedingt eine erfolgreiche Kontextsteuerung auf Seiten des steuernden Systems eine Selbststeuerung auf Seiten des zu steuernden Systems. Inwieweit ist aber die Selbststeuerung des steuernden Systems selbst betroffen? Wenn Kontextsteuerung eben nicht mehr auf unilinearer Kausalität, sondern auf einer Wechselseitigkeit beruht (vgl. Jarren/Röttger 22009: 39), dann implizieren Kontextsteuerungen immer auch Selbststeuerungen eines Unternehmens. Wenn eine Regierung mit einem finanziellen Anreizsystem Naturschutz belohnen und fehlenden Naturschutz bestrafen will, so ist dies einerseits eine versuchte Kontextsteuerung gegenüber der Wirtschaft, andererseits aber auch eine Selbststeuerung der Regierung.

Folglich ist jede Kontextsteuerung auch eine Selbststeuerung des steuernden Systems. Kontextsteuerungsversuche der PR sind also zugleich Selbststeuerungen eines Unternehmens. Wenn aber - wie oben erläutert - unterschieden wird zwischen PR- und unternehmerischen Selbststeuerungen, stellen sich insbesondere die folgenden Fragen:

(a) Welche Kontextsteuerungen der PR sind ohne unternehmerische Selbststeuerungen vorstellbar? Letztlich ist dies die Frage nach der Autonomie der PR.

(b) Welche Risiken ergeben sich für ein Unternehmen, wenn PR das „Werkzeug“ unternehmerischer Selbststeuerung nicht zur Verfügung steht? Dies ist die Frage nach den Folgen eines fehlenden Einflusses von PR.

(c) Ist ein weitgehender Verzicht auf Kontextsteuerungen vorstellbar? Dies ist Frage nach der Relevanz von Kontextsteuerungen.

Beginnen wir mit der Frage, (a) welche PR-Kontextsteuerung keine unternebmerische Selbststenerung bedingen. Dazu dürften zum Beispiel Fragen der operativen Umsetzung von Kommunikationsmaßnahmen zur Darstellung der Unternehmenspolitik zählen. In solchen Fällen wird PR in der Regel eine kognitive Erwartungshaltung bei den Bezugs- 
gruppen unterstellen: Die PR sieht also gute Chancen, dass die relevanten Bezugsgruppen durch Kontextsteuerung gewonnen werden. Arbeitsgrundlage hierfür sind zum Beispiel genehmigte Budgets und die inhaltlichen Konzepte bzw. Aussagen, auf die sich PR und Unternehmensleitung in formellen und informellen Prozessen - also früheren unternehmerischen Selbststeuerungen - geeinigt haben. Solche PR-Kontextsteuerungen können als Routinen der PR bezeichnet werden. Sobald sich PR mit ihren Selbstbeschreibungen aber von dieser vereinbarten Grundlage entfernt, steigt das Risiko, dass die kommunizierten Selbstbeschreibungen intern nicht rückverbunden sind - es also einen Widerspruch zum beobachteten Verhalten gibt (vgl. Kieserling 2005).

Wenn ein wachsender Druck einer Bezugsgruppe erwartet wird oder wenn PR auf Bezugsgruppen mit einer normativen Erwaltungshaltung trifft, reichen diese Arbeitsgrundlagen nicht mehr aus. In solchen Fällen kann es entweder weitere Kontextsteuerungsversuche wie kostenintensive Imagekampagnen oder Corporate Citizenship-Aktivitäten geben, die beide der Zustimmung der Unternehmensleitung bedürfen und damit zugleich Selbststeuerungen sind. Oder der Druck ist so groß geworden, dass weiter reichende unternehmerische Selbststeuerungen wie die Umstellung auf umweltfreundlichere Produktionsverfahren notwendig sind.

Wenn PR die Unterscheidung von Selbststeuerung und Kontextsteuerung managt, impliziert dies, dass es beide Seiten der Unterscheidung braucht. Welche Folgen hat es, wenn PR eine Seite der Unterscheidung fehlt? Eine (b) feblende unternebmerische Selbststeuerung läge vor, wenn eine Unternehmensleitung sich Empfehlungen der PR gänzlich verweigern würde. PR hätte damit zur Legitimation allein das „Werkzeug“ einer sehr beschränkten, weil auf operative Fragen beschränkten Kontextsteuerung. Kurzfristig und in Einzelfällen kann dies durchaus sehr erfolgreich sein. Langfristig droht PR, damit nur noch die Rolle eines „Reparaturbetriebs“ (Liebl 2000: 128) auszufüllen, der durch öffentliche Erklärungen die immer größer werdenden Widersprüche zwischen Unternehmen und ihren relevanten Bezugsgruppen zu „kitten“ versucht. In diesem Fall wäre PR nur noch das Sprachrohr einer lernunwilligen Unternehmensleitung. Solche Unternehmen laufen Gefahr, Kritik relevanter Bezugsgruppen so lange zu unterschätzen, bis der öffentliche Druck so groß geworden ist, dass sehr große Änderungen der Unternehmenspolitik erforderlich sind.

Hier wird deutlich, wie wichtig PR als unternehmerisches Reflexionszentrum sowie unternehmerische Selbststeuerungen sind. Die Legitimation eines Unternehmens und damit die Sicherung von Handlungsspielräumen sind ohne Anpassungsprozesse und damit unternehmerische Selbststeuerungen nicht vorstellbar. Es ist zu vermuten, dass diese Anpassungsprozesse umso geringer ausfallen und die weiteren Handlungsspielräume umso weniger einschränken, je früher sie stattfinden. Daher wird PR auch zu Recht als „Frühwarnsystem“ (Beger/Gärtner/Mathes 1989: 37) bezeichnet.

Eine (c) feblende unternehmerische Kontextsteuerung kann implizieren, dass ein Unternehmen versucht, sehr frühzeitig latente Konflikte durch eine Änderung der Unternehmenspolitik zu vermeiden, indem es auf kritische Produktionsverfahren oder Produkte verzichtet. Der Vorteil einer solchen Strategie ist es, durch fehlende öffentliche Exponiertheit das Risiko zu verringern, zum Objekt einer kritischen Öffentlichkeit zu werden. Grundsätzlich ist zu vermuten, dass ein Verzicht auf Kontextsteuerung umso vielversprechender ist, je mehr ein Unternehmen die „Informationspolitik“ kontrollieren kann und damit Geheimnisse zurückhalten kann. In Einzelfällen mag dies zu beobachten sein, in der Summe ist es jedoch nur schwer vorstellbar, dass ein Unternehmen gänzlich auf Kontextsteuerungen im Rahmen der PR verzichtet. Stattdessen dürften unternehmerische Selbststeuerungen in der deutlichen Mehrzahl von externer Kontext- 
steuerung „begleitet“ werden - Änderungen der Unternehmenspolitik also öffentlich kommuniziert werden.

PR ist also auf beide Seiten der Unterscheidung angewiesen. Und ohne in die ethisch begründete Normativität von Grunig et al. und Cutlip/Center/Broom (61985) zurückzufallen, ist zu vermuten, dass PR-Aktivitäten mit einer Verbindung von Selbststeuerung und Kontextsteuerung im Sinne der Legitimation in der Regel höchst erfolgreich sind. Das hat in der PR vor langer Zeit bereits Zedtwitz-Arnim mit seinen PR-Klassiker „Tu Gutes und rede darüber“ (1961) erkannt. So ist der PR-Alltag heute von vielfältigen symbolischen Selbststeuerungsentscheidungen geprägt. Als symbolische Selbststeuerungsentscheidungen sollen solche Entscheidungen bezeichnet werden, deren (monetäres) Ausmaß im Unternehmen eher gering ist und die vor allem mit Blick auf die öffentliche Wirkung hin getroffen werden. Dazu können zum Beispiel zahlreiche sogenannte „Corporate Social Responsibility“-Programme von Unternehmen gezählt werden, die das nachhaltige und mithin verantwortungsbewusste Wirtschaften verdeutlichen sollen (vgl. Schranz 2007). Häufig erschöpfen sich solche Programme aber neben einigen wenigen konkreten Projekten im Rahmen der Selbststeuerung vor allem auf eine begleitende externe Kommunikation im Rahmen der Kontextsteuerung. Ein ähnliches Verhältnis von Selbststeuerung und Kontextsteuerung ist häufig bei Projekten zur Förderung regenerativer Energien einiger Energie-Unternehmen zu beobachten, das von $\mathrm{Na}-$ turschutzverbänden als "Greenwashing“ kritisiert wird, weil den großen öffentlichen Kommunikationskampagnen nur eine kleine Anzahl an konkreten Projekten gegenübersteht. Die Inszenierung von Lernwilligkeit kann also eine Strategie der Kontextsteuerung sein, hinter der sich eine höchst lernunwillige Unternehmensleitung zu verstecken versucht, um so weiterzumachen wie bisher.

Damit beinhalten symbolische Selbststeuerungsentscheidungen ein langfristiges Risiko für die PR. Denn hier ist man bei der Frage nach der Fiktionalität der PR-Selbstbeschreibungen angelangt. Der hohe reflexive Charakter der Legitimation und der kollektive Bezug verbunden mit einer zunehmenden Fiktionalisierung im Rahmen der Mediengesellschaft machen die herausragende Bedeutung der Selbstdarstellungen deutlich. Die Öffentlichkeit ist für die PR so wichtig, weil sie für viele bei der Zuschreibung von Legitimität so wichtig ist. Aber gerade weil die Gesellschaft sich auf die Nichtnachprüfbarkeit von Behauptungen eingerichtet hat (vgl. Merten 1999), ist sie sich auch ihrer eigenen Verletzbarkeit bewusst und entzieht Vertrauen und Legitimität umso mehr, je mehr sie sich arglistig getäuscht fühlt. Das macht das Risiko deutlich, wenn die kommunizierten Selbstbeschreibungen intern nicht rückverbunden sind (Kieserling 2005). Dieses Risiko gilt gleichermaßen für „ertappte Lügner“ wie für symbolische Selbststeuerungsentscheidungen, die insbesondere langfristig das Vertrauen in PR schwächen.

Dies zeigt deutlich, dass PR zwar auf beide Seiten der Unterscheidung angewiesen ist, aber dennoch kein Zweifel daran besteht, dass die externe Kontextsteuerung immer die präferierte Seite der Unterscheidung ist. Eine Erhöhung der Budgets oder gar eine Änderung der Unternehmenspolitik zur „Befriedung der Umwelt“ dürfte von vielen Unternehmensleitungen schon fast als „worst case“ bewertet werden, da es nicht gelingt, die eigenen Interessen durchzusetzen. Je erfolgreicher also die Kontextsteuerung eines Unternehmens, desto weniger ist Selbststeuerung notwendig (vgl. Everett 2000: 317).

\section{Fazit}

Wenn die Vernachlässigung der Selbstdarstellungsleistung aufgegeben wird und die unternehmerische Selbststeuerung als Äquivalent zur Kontextsteuerung gesehen wird, gewinnen für die PR-Forschung neue Fragen ein deutlich größeres Gewicht. 
Während in der PR-Forschung und insbesondere in den empirischen Arbeiten PRProdukte und ihre Wirkungen im Mittelpunkt des Forschungsinteresses standen, lenkt das Management der Unterscheidung von Selbst- und Kontextsteuerung das Forschungsinteresse auf Entscheidungen und Kommunikation in Unternehmen, die bislang weitgehend als „Black Box“ angesehen wurden. Denn ohne ein Verständnis dieser „Black box“ bleibt nicht nur der gesamte Bereich der unternehmerischen Selbststeuerung verborgen, letztlich kann auch die Kontextsteuerung nur vor dem Hintergrund des Managements der Unterscheidung „verstanden“ werden.

Neben den Beziehungen zwischen der PR und der Unternehmensleitung erscheint hier insbesondere die Konzeptualisierung des PR-Entscheidungshandelns relevant zu sein. Manfred Rühl, der Mentor systemisch orientierter Redaktionsforschung, hat diesen Zusammenhang in der Journalistik bereits vor langer Zeit thematisiert: „Redaktionelles Entscheiden, das Recherchieren, Redigieren, Schreiben sowie andere Handlungs- und Kommunikationsprozesse umfasst [...], führt mithin zu Produktionen, die Ergebnisse komplexer interner Entscheidungsprozesse sind.“ (Rühl 1989: 262) In Anlehnung an Manfred Rühl kann das PR-Entscheidungshandeln als Prozess definiert werden, der sich in die Phasen Analyse, Ziel- und Strategie-Formulierung, Entwicklung eines Umsetzungsprogramms sowie der internen oder/und externen Umsetzung und Evaluation gliedern lässt. Das Umsetzungsprogramm können Kontextsteuerungen wie klassische Pressearbeit, Empfehlungen im Rahmen der Selbststeuerung oder eine Mischung aus beiden sein. Das Differenzmanagement von externer Kontext- und unternehmerischer Selbststeuerung ist folglich hier verortet.

Zwar wird die empirische PR-Forschung auch künftig häufig vor verschlossenen Türen stehen oder Beobachter inszenierten PR-Entscheidens sein, aber zumindest in der PR-Theoriebildung kann die interne Seite der Legitimationsproduktion kaum länger unbeobachtet bleiben. Die Beobachtung des PR-Entscheidungshandelns öffnet den Blick für die Kontingenz externer PR-Kommunikationsangebote und trägt dem strategischen Charakter der PR Rechnung, weil sie das Forschungsinteresse auf den Planungsprozess richtet. So erscheint in der PR das Wissen um diese Zusammenhänge noch deutlich wichtiger als zum Beispiel im - nicht strategischen - Journalismus.

\section{Literatur}

Baerns, Barbara (21991): Öffentlichkeitsarbeit oder Journalismus? Zum Einfluß im Mediensystem. Köln.

Barth, Henrike/Donsbach, Wolfgang (1992): Aktivität und Passivität von Journalisten gegenüber Public Relations. In: Publizistik, 37, 2: 151-196.

Beger, Rudolf/Gärtner, Hans-Dieter/Mathes, Rainer (1989): Unternehmenskommunikation. Grundlagen, Strategien, Instrumente. Wiesbaden.

Cutlip, Scott M. /Center, Allen H./Broom, Glen M. (61985): Effective Public Relations. Englewood Cliffs.

Dozier, David M./Grunig, Larissa A. (1992): The Organization of the Public Relations Function. In: Grunig, James E. (Hrsg.): Excellence in Public Relations and Communication Management. Hillsdale. 395-417.

Dozier, David M./Grunig, Larissa A./Grunig, James E. (1995): Manager's Guide to Excellence in Public Relations and Communication Management. Mahwah.

Druwe, Ulrich/Görlitz, Axel (1992): Politikfeldanalyse als mediale Steuerung. In: Bußhoff, Heinrich (Hrsg.): Politische Steuerung: Steuerbarkeit und Steuerungsfähigkeit. Beiträge zur Grundlagendiskussion. Baden-Baden. 143-164.

Eisenegger, Mark (2005): Reputation in der Mediengesellschaft. Konstitution - Issues Monitoring - Issues Management. Wiesbaden. 
Everett, James L. (2000): Public Relations and the Ecology of Organizational Change. In: Heath, Robert L. (Hrsg.): Handbook of Public Relations. Thousand Oaks: 311-320.

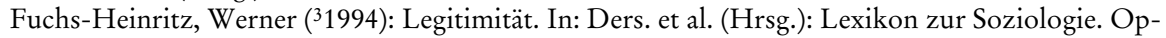
laden. 396.

Gerhards, Jürgen/Neidhardt, Friedhelm (1991): Strukturen und Funktionen moderner Öffentlichkeit: Fragestellungen und Ansätze. In: Müller-Dohm, Stefan/Neumann-Braun, Klaus (Hrsg.): Öffentlichkeit, Kultur, Massenkommunikation. Beiträge zur Medien- und Kommunikationssoziologie. Oldenburg. 35-89.

Görke, Alexander (1999): Risikojournalismus und Risikogesellschaft. Sondierung und Theorieentwurf. Opladen, Wiesbaden.

Grunig, James E./Grunig, Larissa A. (1992): Models of Public Relations and Communication. In: Grunig, James (Hrsg.): Excellence in Public Relations and Communication Management. Hillsdale: $285-325$.

Grunig, James E./Grunig, Larissa A. (2008): Excellence Theory in Public Relations. Past, Present an Future. In: Zerfaß, Ansgar/van Ruler, Betteke/Sriramesh, Krishnamurthy (Hrsg.): Public Relations Research. European an International Perspectives and Innovations. Wiesbaden: 327-347.

Hoffjann, Olaf (2007): Journalismus und Public Relations. Ein Theorieentwurf der Intersystembeziehungen in sozialen Konflikten. Wiesbaden.

Hundhausen, Carl (1951): Werbung um öffentliches Vertrauen. Public Relations. Essen.

Jarren, Otfried/Röttger, Ulrike (22009): Steuerung, Reflexierung und Interpenetration: Kernelemente einer strukturationstheoretisch begründeten PR-Theorie. In: Röttger, Ulrike (Hrsg.): Theorien der Public Relations. Grundlagen und Perspektiven der PR-Forschung. Wiesbaden: 29-49.

Kieserling, André (2005): Selbstbeschreibung von Organisationen. Zur Transformation ihrer Semantik. In: Jäger, Wieland/Schimank, Uwe (Hrsg.): Organisationsgesellschaft. Facetten und Perspektiven. Wiesbaden: 51-88.

Kohring, Matthias/Hug, Detlef Matthias (1997): Öffentlichkeit und Journalismus. Zur Notwendigkeit der Beobachtung gesellschaftlicher Interdependenz - Ein systemtheoretischer Entwurf. In: Medien Journal, 21, 1, 15-33.

Kunczik, Michael (42002): Public Relations. Konzepte und Theorien. Köln.

Kussin, Matthias (2006): Public Relations als Funktion moderner Organisation. Soziologische Analysen. Heidelberg.

Kussin, Matthias (2009): PR-Stellen als Reflexionszentren multireferentieller Organisationen. In: Röttger, Ulrike (Hrsg.): Theorien der Public Relations. Grundlagen und Perspektiven der PRForschung. Wiesbaden: $117-133$.

Langenbucher, Wolfgang R. (1997): WIR sind die KommunikatorInnen! Zu einigen Scheuklappen der Journalismusforschung. In: Bentele, Günter/Haller, Michael (Hrsg.): Aktuelle Entstehung von Öffentlichkeit. Akteure, Strukturen, Veränderungen. Konstanz: 19-38.

Liebl, Franz (2000): Der Schock des Neuen. Entstehung und Management von Issues und Trends. München.

Long, Larry W./Hazleton Jr., Vincent (1993): Definition and Model of the Public Relations Process. In: Fischer, Heinz-Dietrich/Wahl, Ulrike G. (Hrsg.): Public Relations/Öffentlichkeitsarbeit. Geschichte, Grundlage, Grenzziehungen. Frankfurt am Main. 223-236.

Luhmann, Niklas (1981): Ausdifferenzierung des Rechts. Frankfurt am Main.

Merten, Klaus (1999): Einführung in die Kommunikationswissenschaft. Band 1: Grundlagen der Kommunikationswissenschaft. Münster.

Merten, Klaus (2009): Zur Theorie der PR-Theorien. Oder: Kann man PR-Theorien anders als systemisch modellieren? In: Röttger, Ulrike (Hrsg.): Theorien der Public Relations. Grundlagen und Perspektiven der PR-Forschung. Wiesbaden: 51-70.

Merten, Klaus/Westerbarkey, Joachim (1994): Public Opinion und Public Relations. In: Merten, Klaus/Schmidt, Siegfried J./Weischenberg, Siegfried (Hrsg.): Die Wirklichkeit der Medien. Eine Einführung in die Kommunikationswissenschaft. Opladen. S. 188-211.

Metzler, Maribeth S. (2000): The Centrality of Organizational Legitimacy to Public Relations Practice. In: Heath, Robert L. (Hrsg.): Handbook of Public Relations. Thousand Oaks: 321-334. 
Meyer, John W./Rowan, Brian (1977): Institutionalized Organizations: Formal Structure as Myth and Ceremony. In: American Journal of Sociology, 83, 2: 340-363.

Nothhaft, Howard/Wehmeier, Stefan (2009): Vom Umgang mit Komplexität im Kommunikationsmanagement. Eine soziokybernetische Rekonstruktion. In: Röttger, Ulrike (Hrsg.): Theorien der Public Relations. Grundlagen und Perspektiven der PR-Forschung. Wiesbaden: 151-171.

Oeckl, Albert (1964): Handbuch der Public Relations. Theorie und Praxis der Öffentlichkeitsarbeit in Deutschland und der Welt. München.

Oeckl, Albert (1976): PR-Praxis. Der Schlüssel zur Öffentlichkeitsarbeit. Düsseldorf.

Röttger, Ulrike (2000): Public Relations - Organisation und Profession. Öffentlichkeitsarbeit als Organisationsfunktion. Eine Berufsfeldstudie. Wiesbaden.

Röttger, Ulrike (2008): Public Relations. In: Bruhn, Manfred/Esch, Franz-Rudolf/Langner, Tobias (Hrsg.): Handbuch Kommunikation. Grundlagen, innovative Ansätze, praktische Umsetzungen. Wiesbaden: 67-83.

Röttger, Ulrike (2009): Welche Theorien für welche PR? In: Dies. (Hrsg.): Theorien der Public Relations. Grundlagen und Perspektiven der PR-Forschung. Wiesbaden: 10-25.

Röttger, Ulrike/Hoffmann, Jochen/Jarren, Otfried (2003): Public Relations in der Schweiz. Eine empirische Studie zum Berufsfeld Öffentlichkeitsarbeit. Konstanz.

Rolke, Lothar (1999): Die gesellschaftliche Kernfunktion von Public Relations - ein Beitrag zur kommunikationswissenschaftlichen Theoriediskussion. In: Publizistik, 44, 4: 431-444.

Ronneberger, Franz (1977): Legitimation durch Information. Düsseldorf, Wien.

Ronneberger, Franz/Rühl, Manfred (1992): Theorie der Public Relations. Ein Entwurf. Opladen.

Rühl, Manfred (1989): Organisatorischer Journalismus. Tendenzen der Redaktionsforschung. In: Kaase, Max/Schulz, Winfried (Hrsg.): Massenkommunikation. Theorien, Methoden, Befunde. Opladen. 253-269.

Ruß-Mohl, Stephan (1992): Gefährdete Autonomie? Zur Außen- und Selbststeuerung von Public Relations - Das Beispiel USA. In: Avenarius, Horst/Armbrecht, Wolfgang (Hrsg.): Ist Public Relations eine Wissenschaft? Eine Einführung. Opladen: 311-324.

Schranz, Mario (2007): Wirtschaft zwischen Profit und Moral. Die gesellschaftliche Verantwortung von Unternehmen im Rahmen der öffentlichen Kommunikation. Wiesbaden.

Szyszka, Peter (2004): PR-Arbeit als Organisationsfunktion. Konturen eines organisationalen Theorieentwurfs zu Public Relations und Kommunikationsmanagement. In: Röttger, Ulrike (Hrsg.): Theorien der Public Relations. Grundlagen und Perspektiven der PR-Forschung. Wiesbaden: $149-168$.

Szyszka, Peter (2009): Organisation und Kommunikation: Integrativer Ansatz einer Theorie zu Public Relations und Public Relations-Management. In: Röttger, Ulrike (Hrsg.): Theorien der Public Relations. Grundlagen und Perspektiven der PR-Forschung. Wiesbaden: 135-150.

Szyszka, Peter/Bentele, Günter (2005): Legitimität/Legitimation. In: Bentele, Günter/Fröhlich, Romy/Szyszka, Peter (Hrsg.): Handbuch der Public Relations. Wissenschaftliche Grundlagen und berufliches Handeln. Mit Lexikon. Wiesbaden: $592 \mathrm{f}$.

Teubner, Gunther/Willke, Helmut (1984): Kontext und Autonomie: Gesellschaftliche Selbststeuerung durch reflexives Recht. In: Zeitschrift für Rechtssoziologie, 6, 1: 4-35.

Willke, Helmut (1992): Beobachtung, Beratung und Steuerung von Organisationen in systemtheoretischer Sicht. In: Wimmer, Rudolf (Hrsg.): Organisationsberatung. Neue Wege und Konzepte. Wiesbaden: 17-42.

Willke, Helmut (21993): Systemtheorie entwickelter Gesellschaften. Dynamik und Riskanz moderner gesellschaftlicher Selbstorganisation. Weinheim, München.

Willke, Helmut (31995): Systemtheorie III: Steuerungstheorie. Stuttgart.

Willke, Helmut (1996): Ironie des Staates. Grundlinien einer Staatstheorie polyzentrischer Gesellschaft. Frankfurt am Main.

Willke, Helmut (1997): Supervision des Staates. Frankfurt am Main.

Zedtwitz-Arnim, Georg-Volksmar Graf von (1961): Tu Gutes und rede darüber. Berlin.

Zerfaß, Ansgar (2004): Unternehmensführung und Öffentlichkeitsarbeit: Grundlegung einer Theorie der Unternehmenskommunikation und Public Relations. Wiesbaden. 